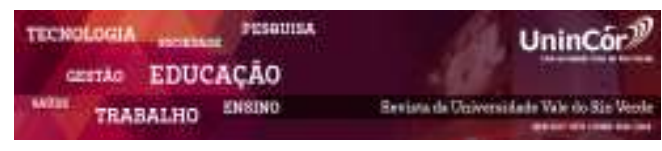

Revista da Universidade Vale do Rio Verde ISSN: 1517-0276 / EISSN: 2236-5362 Vol. 16 | n. 1 | Ano 2018

Lauana Aparecida Santos Universidade Federal de Alfenas e Centro Universitário do Sul de Minas Lauanasantos20@gmail.com

Janaina Aparecida Ruella Universidade Federal de Alfenas jjana564@gmail.com

\section{CONTRIBUIÇÃO DA METODOLOGIA DE TRABALHOS DE EXTENSÃO NA FORMAÇÃO DE PROFISSIONAIS DA SAÚDE: da teoria para a prática}

\section{RESUMO}

A extensão universitária auxilia no caminho para o desenvolvimento de uma formação acadêmica. Esses trabalhos acabam por assumir novas percepções e concepções, no qual a comunidade passa ser participativa e ajuda na construção do conhecimento do próprio docente e do acadêmico. O objetivo desta revisão foi abordar a importância da metodologia de trabalhos de extensão na formação de profissionais da área da saúde dentro de uma Universidade. Para isto foram consultados artigos a partir de uma busca utilizando as plataformas de pesquisa PubMed, Scielo, Lilacs e Cochrane e utilizado como descritores extensão universitária, acadêmicos da saúde, educação e relação comunidadeuniversidade, sem restrição de data de publicação. Foi possível observar a importância da utilização de projetos de extensão na formação de profissionais da saúde. A partir destes trabalhos foi possível observar os aspectos positivos no ensino destes profissionais. A partir desta revisão conclui-se que os projetos de extensão são fundamentais para a formação de profissionais humanistas e generalistas.

Palavras-chave: Extensão universitária. Acadêmicos da saúde. Educação e relação comunidade-universidade.

\section{CONTRIBUTION OF THE METHODOLOGY OF EXTENSION WORK IN THE TRAINING OF HEALTH PROFESSIONALS: from theory to practice}

\begin{abstract}
The university extension helps in the way for the development of an academic formation. These works end up assuming new perceptions and conceptions, in which the community happens to be participatory and helps in the construction of the knowledge of the own teacher and the academic. The purpose of this review was to address the importance of the methodology of extension work in the training of health professionals within a University. For this, articles were searched from a search using the PubMed, Scielo, Lilacs and Cochrane research platforms and used as university extension descriptors, health academic, education and communityuniversity relationship, without publication date restriction. It was possible to observe the importance of the use of extension projects in the training of health professionals. From these works it was possible to observe the positive aspects in the teaching of these professionals. From this review it is concluded that extension projects are fundamental for the training of humanistic and generalist professionals.
\end{abstract}


Keywords: University extension. Academics of health. Education and community-university relationship.

Recebido em: 12/12/2017 - Aprovado em: 03/03/2018 - Disponibilizado em: 15/07/2018

\section{INTRODUÇÃO}

Os trabalhos de extensão universitária auxiliam em um dos caminhos para o desenvolvimento de uma formação acadêmica. Por meio, destes trabalhos é possível integrar a teoria e a prática. Esta integração possibilita uma comunicação com a sociedade e também uma troca de saberes. Desse modo, acaba acontecendo uma socialização e construção de novos conhecimentos a partir da prática. A sociedade se beneficia, bem como também, o próprio discente acaba ganhando mais habilidades e abrindo a mente para um cenário criativista e humanista (MANCHUR, SURIANI; CUNHA, 2013).

O estabelecimento de um currículo que seja inovador faz com que os educadores compreendam que o modelo tradicional de ensino, somente fundamentado na doença e transmissão dos conhecimentos acaba por afetar a visão do discente com a prática da saúde. Conceitos e enfoques pedagógicos afirmam que são necessários aspectos formativos e uma maior prática dos conhecimentos adquiridos (MITRE et al., 2008).

Os projetos de extensão universitária acabam por assumir novas percepções e concepções, no qual a comunidade passa a ser mais participativa, bem como ajuda na construção do conhecimento do próprio docente e também o acadêmico, por meio destas atividades. Todos acabam por desenvolver um senso crítico de acordo com a realidade (MANCHUR, SURIANI; CUNHA, 2013).

Considerando os aspectos positivos apresentados pelos projetos de extensão, o objetivo deste artigo é conhecer ainda mais sobre estes quando se trata de profissionais da área da saúde e abordar os aspectos positivos desta metodologia de trabalho com enfoque nestes cursos de graduação. Dessa forma, é importante esclarecer que a formação de um profissional da área da saúde precisa ser humanista, ou seja, conciliando a prática com a teoria e onde possa ter mais contato com os possíveis pacientes. Os objetivos deste estudo foram abordar os aspectos positivos desta metodologia de trabalho com enfoque nos cursos de graduação da área da saúde. Descrever os benefícios desta prática educacional para sociedade e na formação de um profissional da saúde humanista.

\section{MÉTODOS}

Este estudo foi uma revisão de literatura científica não sistemática (GIL, 1994). Na execução foram consultados artigos a partir de uma busca utilizando as plataformas de pesquisa Scielo, Lilacs e Cochrane. Foram utilizados como descritores; extensão universitária, acadêmicos da saúde, educação e relação 
comunidade-universidade, sem restrição de data de publicação. Foram incluídos os artigos que abordaram o papel da extensão universitária na vida acadêmica dos graduandos da área da saúde e os contextos históricos desta metodologia de ensino. Os artigos encontrados foram selecionados de acordo com o assunto abordado. Durante a busca também foram incluídos citações citados nas referências dos artigos selecionados de acordo com os critérios estabelecidos.

\section{REVISÃO DE LITERATURA}

\section{A extensão universitária}

Na Inglaterra foi onde surgiu a extensão universitária. Sendo possível observar que a criação foi em decorrência da necessidade advinda da Revolução Industrial em conjunto com o capitalismo. Neste momento, embora a função da Universidade fosse à formação quase que exclusiva da elite era possível perceber a existência de uma preocupação em levar informações por meios dos cursos de graduação às classes populares (MANCHUR， SURIANI; CUNHA， 2013; RODRIGUES, 1997).

A prática de projetos de extensão aos poucos foi sendo disseminada pelas instituições tanto europeias e norte-americanas que ofertavam cursos técnicos e profissionalizantes. Já no Brasil a extensão universitária foi sendo trabalhada de acordo com os objetos impostos por poderes públicos, sociais e econômicos. De acordo, com Machur, Suriani e Cunha (2013) e Rodrigues (1997), a Universidade era submissa às regulamentações do Estado e voltada à formação de classes sociais mais altas brasileiras. Des- se modo, as classes populares não tinham acesso a este tipo de ensino. Então neste período as práticas extensionistas visavam apenas os cursos técnicos objetivando a capacitação de mão de obra para o trabalho. Na década de 80 este cenário começa a mudar e onde a Universidade passou a ter mais autonomia e onde foi necessária uma maior aproximação com a sociedade em diferentes escalas de classes sociais (MANCHUR, SURIANI; CUNHA, 2013).

Neste sentido esta metodologia passa assumir novas percepções, onde a comunidade deixa de ser passiva e começa a ser mais participante. Este tipo de metodologia além de contribuir para o conhecimento do professor também ajuda na do aluno, podendo desenvolver senso crítico sobre as suas pesquisas e com a própria realidade.

\section{Metodologias ativas: Fundamentos teóricos}

A formação dos profissionais da área da saúde historicamente tem sido pautada utilizando metodologias que são tradicionais. No qual o processo de ensino-aprendizagem tem sido meramente à reprodução do conhecimento, onde o docente assume o papel de transmissor de conteúdo e onde o discente faz a retenção e a repetição, possibilitando que se torne sem crítica e reflexão (MITRE et al., 2008).

Atualmente, no contexto social os meios comunicativos estão favorecendo o avanço das novas tecnologias e a percepção do mundo, promovendo uma rede de relação dinâmica. Na formação de profissionais da saúde surgem questionamentos sobre como deve ser o perfil destes formandos, preocupando-se à tendência à especialização precoce e ao ensino marcado por parâ- 
metros curriculares. Nos primeiros anos de formação básica são enfatizados no ensino centrado no ambiente hospitalar, com enfoque na atenção curativa, individual da doença, tendo como a produção de um ensino dissociado do serviço das reais necessidades dos sistemas de saúde (COLL, 2000; MITRE et al., 2008) .

O surgimento no cenário da educação superior da Lei de Diretrizes e Bases da Educação Nacional - LDBEN define o estímulo ao conhecimento dos problemas sociais atuais e como deve ser a prestação de serviços especializados à população. Houve uma reafirmação destas prerrogativas pelas Diretrizes Curriculares para grande parte dos cursos da área de saúde, destacando a importância do atendimento às demandas sociais em destaque para o Sistema Único de Saúde (DEMO, 2004).

A partir deste momento as instituições são solicitadas a mudarem suas práticas pedagógicas, objetivando se aproximarem da realidade social e de motivarem o corpo docente e também dos discentes a obterem novas redes de aprendizado. Considerando que a graduação possui duração de alguns anos e a prática profissional pode durar por décadas. Os conhecimentos e competências vão transformando velozmente, tornando fundamental pensar em metodologias de ensino em uma prática que seja libertadora, na formação destes profissionais (MITRE et al., 2008).

\section{Relação Universidade e comunidade}

As Instituições de Ensino Superior desde sua origem tem como princípios ser inovadora, inquietadora e revolucionária. Observam-se a- vanços inestimáveis da sociedade, sendo desse modo fundamental resgatar a função primordial das universidades e seu caráter civilizatório, no qual promove valores culturais, morais e intelectuais (NUNES e SILVA, 2011).

A reflexão sobre o papel social que é desempenhado pelas universidades em especial sobre o cumprimento da função da produção de conhecimentos, social e cientificamente relevante, é necessário tornar acessível a todos. Nota-se que as atividades de extensão deve ser um dos principais componentes quanto a função do ensino superior atualmente, no qual, quando estas necessidades da atuação da universidade na comunidade forem percebidas, já estarão cumprindo as suas funções (SILVA, 1997).

$\mathrm{Na}$ extensão universitária, ocorre uma troca de conhecimentos em que a universidade também aprende com a comunidade sobre seus valores e cultura. Assim, a universidade pode planejar e executar as atividades de projetos voltados à comunidade respeitando e não violando estes valores e culturas. Também pode ocorrer uma troca de valores entre a universidade e o meio social (NUNES e SILVA, 2011).

Portanto, através dos projetos de extensão a universidade tem oportunidade de levar à comunidade os seus conhecimentos que é detentora, sendo estes conhecimentos produzidos pela pesquisa científica. Esta se torna, na qual a universidade social e democratizadora do conhecimento, levando aqueles que não são acadêmicos universitários. Contudo, o conhecimento não se torna um privilégio de poucos, mas sendo este difundido pela comunidade (SILVA, 1997).

A instituição de ensino superior, ao comunicar-se com a realidade da sociedade local, 
regional e/ou nacional, tem a possibilidade de renovar-se constantemente e conduzindo para um atendimento da verdadeira realidade do país (NUNES e SILVA, 2011).

\section{Vantagens dos projetos de extensão aos aca- dêmicos}

Segundo Biscarde e coloboradores (2014), um projeto realizado foi avaliado de forma positiva pelos discentes, supervisores docentes e também gestores. No qual, tendo um consenso na importância da ampliação do processo formativo dos acadêmicos. Descrevendo que os projetos de extensão contribuíram para potencializar a construção de indivíduos sociais ou coletivos, a partir das vivencias desencadeadas durante os trabalhos. Revelando principalmente como uma transformação na visão de mundo e na maneira como trabalhar e no relacionamento com os serviços de saúde.

Concluindo que as principais vantagens são:

- Ampliar o percurso e o processo formativo dos alunos.

- Desenvolver atividades de pesquisa articuladas com os projetos de extensão.

- Promoção da integração entre estudantes, gestores, técnicos e profissionais do sistema municipal de saúde.

- A ampliação do projeto ser possível e do desenvolvimento de programas de vivencias extensionistas na universidade.

- Possibilidade da inclusão das discussões sobre as propostas de flexibilização curricular, que ocorrem entre as reuniões de colegiado de curso e dos centros acadêmicos.
Desse modo, é possível observar as várias vantagens de trabalhos voltados à comunidade e o retorno positivo por parte dos alunos (BISCARDE et al., 2014).

\section{Os projetos de extensão como um dos elemen- tos de formação universitária da saúde}

A interdisciplinaridade entre ensino, pesquisa e projetos de extensão é atualmente um dos desafios das universidades. Nota-se que enquanto a pesquisa científica e o ensino tem sido alvo de discussões que originaram elaborados sistemas de avaliação da produção científica e da qualidade dos cursos de graduação, porém a extensão universitária não recebeu a mesma atenção e muito menos sofreu as transformações que são necessárias para acompanhar a evolução do ensino superior. Promover projetos de extensão ainda é um desafio para muitos docentes e discentes que queiram realizar esta atividade dentro da universidade. Falta de incentivo por parte de setores administrativos e de coordenação, torna este trabalho dispendioso e burocrático o que acaba por desmotivar a todos (SILVA e VASCONCELOS, 2006).

A criação de ligas acadêmicas é um passo fundamental para o discente ter os primeiros contatos com a vida de extensionista e poder experimentar as experiências que a comunidade pode oferecer. O Projeto Rondon também é uma destas ferramentas de formação e foi desenvolvido pelo Ministério da Defesa, em parceria com os governos do estado, município e com as universidades públicas e privadas. Este tipo de trabalho tem como objetivo contribuir para a formação do jovem universitário de diversos cursos 
de graduação como cidadão para o desenvolvimento sustentável e nas comunidades carentes. Este tipo de trabalho é uma ferramenta poderosa de transformação, pois permite que as ações tenham efeitos duradouros na comunidade e ao longo prazo favorecendo a população, economia, meio ambiente e administração. Neste momento, aprimora os valores humanitários e a responsabilidade social e coletiva (SILVA e VASCONCELOS, 2006).

As feiras de saúde também é um passo de contato com a comunidade e modelo prático para a aplicação de todos os conhecimentos adquiridos na sala de aula. O docente também vai a campo e onde pode abrir a sua mente para novas ideias voltadas a comunidade.

\section{O profissional da saúde, a organização do en- sino e do trabalho}

O profissional de saúde em sua formação é necessário conhecimento científico e tecnológico, no entanto é importante o conhecimento de natureza humanística e social relativo ao processo de cuidar, desenvolver projetos terapêuticos singulares e na formulação e avaliação de políticas e coordenação de conduzir serviços de saúde. Somente o diploma não é suficiente para garantir a qualificação, é necessária sempre uma atualização profissional. O desenvolvimento do ato de cuidar deve envolver o contato com o paciente ou com o outro e assim, aprendendo o relacionamento com o próximo. No qual, com o dia a dia do trabalho possa minimizar as possíveis dificuldades a serem enfrentadas (CANESQUI, 2006).

$\mathrm{Na}$ formação do profissional da saúde é frequente que as disciplinas biológicas (anatomi- a, fisiologia e outras) sejam as primeiras do curso e isto tem um significado na formação destes profissionais. A maioria dos cursos passa a maior parte apresentando os seus conteúdos voltados simplesmente para o corpo e não abordando a história de vida e realidade social ao qual aquele indivíduo tenha passado ou está passando. Onde acaba formando profissionais não voltados às atividades e necessidades da comunidade. Com base em uma nova restruturação e aplicação de projetos sociais/extensionistas voltados a comunidades estes profissionais não existiram mais, isto faz com que todos saiam ganhando (CAMARGO, 2003).

\section{A Saúde coletiva e a relevância na formação social}

Estudiosos da saúde publica como Oswaldo Cruz e Carlos Chagas foram os primeiros a voltar sua preocupação com a saúde coletiva e onde começou trabalhos intensivos na promoção da saúde. Trabalhos como estes reforçaram a importância da cobertura dos cuidados primários a população tanto de periferia urbana como também a rural. A partir, destas práticas foi possível expandir os conhecimentos sobre a educação popular e onde também expandiram as bases universitárias para o movimento de reforma sanitarista (CECCIM, 2004).

A atenção básica a saúde contribui para atividades de prevenção, onde tornam os custos a saúde menores, facilita o acesso a saúde a todos e como a melhora na qualidade de vida da população. Desse modo, este contato dos discentes com a comunidade abrange em todos os aspectos os benéficos na formação de um profissional e 
ainda principalmente quando se trata de profis-

\section{RESULTADOS E DISCUSSÃO}

A partir da leitura e estabelecidos critérios de seleção para a construção da revisão de literatura foi incluídos 13 estudos. Destes estudos 8 abordavam a importância da aplicação de projetos de extensão em cursos da área da saúde. Realizando comparações entre os estudos todos foi observado que os projetos de extensão tem sido como uma estratégia para a formação de profissionais da saúde. Também foi possível observar pontos positivos do contato dos graduandos com a sociedade.

Segundo Da Silva, 2008 há muitos desafios para transformar a sociedade, é a partir da educação que pode ser minimizados estes problemas. A Universidade voltada à comunidade não deixa de garantir a formação de profissionais com as devidas capacidades técnicas. Após, atividades como estas reforçam ainda mais os conhecimentos aprendidos dentro da sala de aula.

Portanto dos estudos consultados é possível observar que todos apresentam aspectos positivos do trabalho com projetos de extensão. Vale ressaltar que os estudos abordam a importância do papel do docente na participação conjunta com estes projetos. Outro aspecto abordado é a importância destes trabalhos no estimulo pela busca do conhecimento para que a informação seja transmitida de forma correta.

A realidade vivida demonstra que a partir dos estudos não se pode ignorar os problemas da sociedade principalmente quando se trata da saúde coletiva. Faz-se importante informar a sociedade sobre as possíveis doenças transmitidas e sionais da área da saúde (CARVALHO, 2001).

como trata-las, evitar contaminação e possíveis sintomas. Então é possível observar que levar a prática de alunos de cursos da saúde a partir de projetos de extensão, Rondom e feiras de saúde estas informações podem ser transmitidas mais facilmente e onde observa-se uma diminuição da incidência de uma determinada doença.

A formação dos profissionais da área da saúde precisa ser generalista e humanista. As diferentes transformações que dinamizam o conhecimento e acontecimentos pelo mundo coloca em xeque as verdades e estimula a curiosidade. Então a utilização como metodologia ativa os projetos de extensão irá promover um profissional capaz de lidar com diferentes situações e estimular a buscar novas alternativas para a solução de problemas sociais.

\section{CONCLUSÃO}

Diante dos estudos realizados é possível concluir que a universidade tem um papel fundamental na construção da sociedade. É de fundamental importância à prática de projetos de extensão e feiras de saúde na comunidade para a construção e formação de um profissional da área da saúde com responsabilidade e cidadania.

$\mathrm{O}$ corpo docente tem um papel fundamental no estímulo destes estudantes e por onde começa a troca de conhecimentos e interação. Dessa forma a sociedade acaba por ajudar na interação e ensino destes profissionais. Portanto o uso de projetos de extensão na formação destes profissionais possibilita que todos saiam ganhando. 


\section{REFERÊNCIAS}

BISCARDE, DGS. et al. Formação em saúde, extensão universitária e Sistema Único de Saúde (SUS): conexões necessárias entre conhecimento e intervenção centradas na realidade e repercussões no processo formativo. Comunicação Saúde Educação. 201418(48):177-86.

CAMARGO, Jr., KR Biomedicina, saber \& ciência: uma abordagem crítica. São Paulo: Janeiro: Hucitec, 2003.

CANESQUI, AM. (org.) Dilemas e desafios das ciências sociais na Saúde Coletiva. $2^{\mathrm{a}}$.ed. São PauloRio de Janeiro: Hucitec-ABRASCO, 2006.

CARVALHO, YM Atividade física e saúde: onde está e quem é o "sujeito" da relação? Revista Brasileira de Ciências do Esporte. 2001;22(2);9-21.

CECCIM, RB. Equipe de saúde: a perspectiva entredisciplinar na produção dos atos terapêuticos. In Pinheiro R; Mattos RA. Cuidado: as fronteiras da integralidade. Rio de Janeiro: IMS/Uerj: Abrasco, 2004: 259-278.

COLL, C. Psicologia e currículo: uma aproximação psicopedagógica a elaboração do currículo escolar. São Paulo: Ática; 2000.

DA SILVA, JLM. Contribuição da extensão comunitária para a inserção na atenção básica e formação de profissionais da área da saúde. X Encontro de Extensão, 2008.

DEMO, P. Professor do futuro e reconstrução do conhecimento. Petrópolis: Vozes; 2004.

Hucitec, 2003.

GIL, AC. Métodos e Técnicas de Pesquisa Social. São Paulo, Atlas. 1994.

MITRE, SM. et al. Metodologias ativas de ensinoaprendizagem na formação profissional em saúde: debates atuais. Ciência \& Saúde Coletiva. 2008;13(Sup 2):2133-2144.

NUNES, ALPF; SILVA, MBC. A extensão universitária no ensino superior e a sociedade. MalEstar e Sociedade. 2011:119-133.

OLIVEIRA, FLB; SILVA, JJA. Extensão universitária: contribuições na formação de discentes de Enfermagem. Rev. Bras. Pesq. Saúde, Vitória. 2015;17(1): 19-24.

SILVA, MS; VASCONCELOS, SD. Extensão universitária e formação profissional: avaliação da experiência das Ciências Biológicas na Universidade
Federal de Pernambuco. Estudos em Avaliação Educacional. 2006;17( 33).

SILVA, O. da. O que é extensão universitária. Integração: ensino, pesquisa e extensão, São Paulo. 1997; 3(9):148- 9.

Lauana Aparecida Santos
Mestra em Biociências Aplicadas à Saúde pela
Universidade Federal de Alfenas e Aluna de
Especialização do Curso de Pós-Graduação em
Docência para o Ensino Superior do Centro
Universitário do Sul de Minas - UNIS.

Janaina Aparecida Ruella

Acadêmica do Curso de Letras da Universidade Federal de Alfenas - UNIFAL 